\title{
Analysis of Dynamic Stress into Information Transmission Systems
}

\author{
N. A. Akbarova ${ }^{1}$, V. I. Redkorechev ${ }^{2}$ \\ Department of Geometry, National University of Uzbekistan, Tashkent, Uzbekistan
}

Institute of Applied Physics, National University of Uzbekistan, Talabogarshaharchasi, 3A, Tashkent, 100174, Uzbekistan

\begin{abstract}
In article the diagnostics of optical communication lines developed on the basis of modern information technology achievement of registration and processing of digital information is shown, which is based on a supersensitive method of digital, doubleexposed holographic intreferometry.
\end{abstract}

Keywords: information transmission, optical fibers, digital holographic interferometry

\section{Introduction}

There are a lot of elbows, adapters between the cables of different sections, and connectors with available equipment in the modern fiber-optic data transfer lines and in the local network lines particularly. All this equipment endures by various continuous mechanical traffics and weather exposures, which can bring in distortions and additional noise to a flow of the transmitted information. For qualitative work securing the exact monitoring of fiber-optic data transfer lines is extremely necessary. To the present time the advanced methods of fiber-optic line diagnostics are reduced basically to measurement of losses and are not sensitive to small external perturbation.

\section{Methodological Base}

In our work the diagnostics of optical communication lines developed on the basis of modern information technology achievement of registration and processing of digital information is described. The offered technique is based on a supersensitive method of digital double-exposed holographic intreferometry [1]. In contrast to traditional holography [2,3] where the electromagnetic field wavefront record and reconstruction are occurred in an analog form (using a photoplate, thermoplastic, photorefractive materials), the digital holography is a way of wavefront record in a digital form with the subsequent its retention, measurement, transformation, and reconstruction by mathematical methods in computers [4,5]. In digital holographic interferometry, two wave fronts reconstructed from two digital holograms $\Gamma 1$ and $Г 2$ are compared (numerically interfere). In this case $\Gamma 1$ is the hologram of the unperturbed object and $\Gamma 2$ is the hologram of object with induced optical heterogeneousness. As a result of two wave front summing the interferogram is obtained. It is possible to determine the phase changes arisen at the temporal interval between records of $\Gamma 1$ and $\Gamma 2$ holograms in the investigated objects on basis of analysis of curvatures and locations of fringes at the obtained interferogram. Each digital hologram is recorded in the field of pulse or cw laser radiation using a CCD matrix of the digital chamber. At this case the shot time is specified by digital chamber control gate or laser pulse durations.
The idea of application of computer processing for wavefront reconstruction using the images written down holographically was offered for the first time by J.W.Goodman and R.W.Lawrence[6], and also by R.W.Kronrod with co-workers [7]. The development of information technologies and solid-state radiation detectors has allowed writing down the holograms with the help of photosensitive matrixes on basis of charge-transfer devices (CCD chambers) in a digital form. In these cases the processes of retention and processing of the holograms are completely digital ones and were called as digital holography. The application of digital holography opens great potentialities for qualitative and moreover exact quantitative analysis of object properties such as precision displacement of surface points at the deformation and fault analysis, definition of the object shape, measurement of refraction index in transparent media, study of microparticle trajectories, and so on.

The numerical reconstruction of the hologram written down by a digital image is carried out according to the scalar diffraction theory in the Fresnel approach for the RayleighSommerfeld diffraction integral $[8,9]$. This technique serves as a starting point for numerical reconstruction of the images in the digital holography in paraxial approximation. The resulting field is determined by twofold Fourier transform of intensity distribution product in the obtained hologram. At accounts the quantization is entered taking into account the pixel sizes of a CCD matrix.

\section{Algorithm Description}

In our measurements of induced phase heterogeneities in optical fibers the algorithm of digital intreferogram reconstruction under the recorded holograms was similar to algorithm submitted in [4]. The intensity distribution in a plane of hologram record $I(x, y)$ is determined by summing of a module squares of complex amplitudes of object $\mathrm{O}(\mathrm{x}, \mathrm{y})$ and background $\mathrm{R}(\mathrm{x}, \mathrm{y})$ waves:

$\mathrm{I}(\mathrm{x}, \mathrm{y})=|\mathrm{R}(\mathrm{x}, \mathrm{y})|^{2}+|\mathrm{O}(\mathrm{x}, \mathrm{y})|^{2}+\mathrm{R}(\mathrm{x}, \mathrm{y}) \mathrm{O}(\mathrm{x}, \mathrm{y})+\mathrm{R} *(\mathrm{x}, \mathrm{y}) \mathrm{O}(\mathrm{x}, \mathrm{y})(1)$

The last two terms of the Eq. (1) contain the information appropriate to amplitude and a phase of the object wave. This information can be discriminated by the Fourier 


\section{International Journal of Science and Research (IJSR) \\ ISSN (Online): 2319-7064}

Index Copernicus Value (2013): 6.14 | Impact Factor (2014): 5.611

transform method. If the hologram is recorded by a light source with the restricted output aperture (in our case, the restricted light beam), after transformation we receive a Fourier spectrum of the hologram with four located areas of spatial frequencies, which correspond to the various terms of the Eq. (1). First two terms of Eq. (1) form the zero order of the spectrum, which is located at the centre of twodimensional Fourier plane. The third and fourth terms of Eq. (1) form two connected spectral areas located symmetrically concerning the centre and correspond to the complex amplitude of object wave. If one of these located spectrum areas is separated and then the inverse Fourier transform is applied, the phase front of object wave (real or imaginary ones depending on the chosen separation area) can be reconstructed in the object image plane.

Thus the following algorithm the recording and reconstruction was used. In the beginning the hologram $\Gamma 1$ (non-loaded optical fiber) is recorded, then in the next light pulse the hologram $\Gamma 2$ (loaded optical fiber) is recorded. Then the two-dimensional Fourier spectrum is constructed under the obtained holograms. In this spectrum the area for reconstruction is selected. Under this selected area the digital phase fronts $\Gamma 1$ and $\Gamma 2$ are reconstructed by inverse Fourier transform and their interferogram is calculated. Then the algorithm of a phase unwrapping similar to [4] is used. The real surface of the indignant phase of radiation transmitted through deformable optical fiber is reconstructed.

\section{Experimental}

For record and analysis of induced optical heterogeneities in optical fibers the traditional schemes of Young and Michelson interferometers were used. The interferometer was assembled using two pieces of single-mode fiber for optical telephony. The diameter of fiber core was $125 \mathrm{~mm}$. The lengths of each optical fiber pieces were $2 \mathrm{~m}$. The cable sheath was removed from the fiber input and these cable inputs incorporated together in parallel for input of laser radiation in the interferometer. At the interferometer input the He-Ne laser beam with wavelength of $0.63 \mathrm{~mm}$ and radiation power of $3 \mathrm{~mW}$ are transmitted without focusing optics.

The cable sheath was also removed from the fiber end and these cable ends incorporated together in parallel at the spatial interval. The interferometer optical fibers were rolled by rings with diameter of about $30 \mathrm{~cm}$ and were situated in the free (unfixed) conditions on an optical table.

In such scheme, two optical fibers formed object and background light beams. In Fig.1 and Fig.2 the meter schemes assembled by the Young and Michelson interferometers are shown.

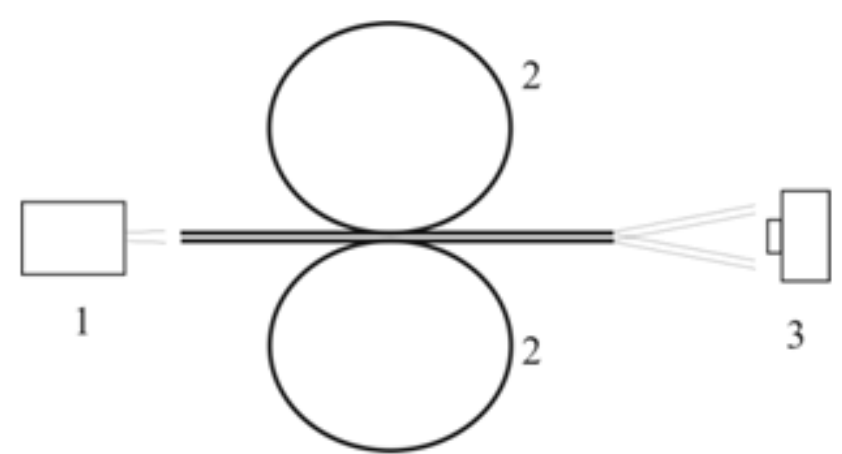

Figure 1: Meter scheme based on the Young interferometer. 1: laser, 2: optical fiber, 3: photographic camera.

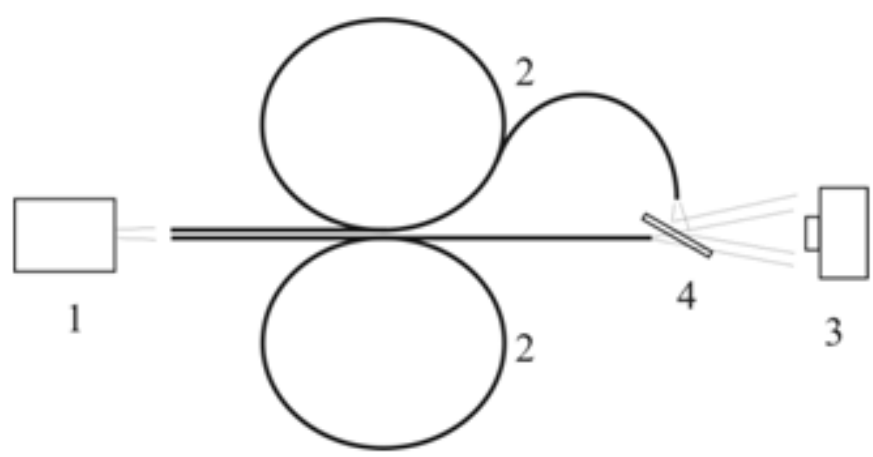

Figure 2: Meter scheme based on the Michelson interferometer. 1: laser, 2: optical fiber, 3: photographic camera, 4: splitter.

The analysis of interferometer response on external influences was carried out by loaded of the first background fiber by paper belt weighing $3 \mathrm{mg}$ or by location at the distance of $10 \mathrm{~cm}$ интерферометра of a heat source with the temperature of $35 \mathrm{C}$ (at the temperature of ambient space of 25 C).

At the interferometer output for digital registration of the holograms formed by interference of background and object beams the monochrome digital photographic camera with the pixel size of $9 \times 9 \mathrm{~mm}$ was used. The record was carried out on the matrix area of $1000 \times 1000$ pixels. Time of exposure was $1 / 10000 \mathrm{sec}$.

\section{Results and Discussion}

The holograms were recorded using the experimental arrangements described above. In Fig. 3 the digital holograms recorded in a condition of interferometer loaded by the paper bent (a) and without loading (b) are presented. The camera objective is removed. The large heterogeneity of intensity is connected with poor-quality chip of the input and output ends of the optical fibers.

In Fig. 4 the digital hologram recorded by camera equipped by objective is shown. The record was carried out under the scheme presented in Fig.2. The objective forms the image of interference pattern at the distance of $5 \mathrm{~mm}$ from the beam splitter.

At the next stage the different edinterferogram was reconstructed under the recorded holograms with the help of algorithm described above. This interferogram contains the quantitative information on phase changes in laser radiation 


\section{International Journal of Science and Research (IJSR) \\ ISSN (Online): 2319-7064 \\ Index Copernicus Value (2013): 6.14 | Impact Factor (2014): 5.611}

transmitted through loaded optical fiber. Then the area shown in Fig.4 by rectangle was selected on this interferogram. In the borders of selected area the surface of phase distortions shown on Fig. 5 was reconstructed.

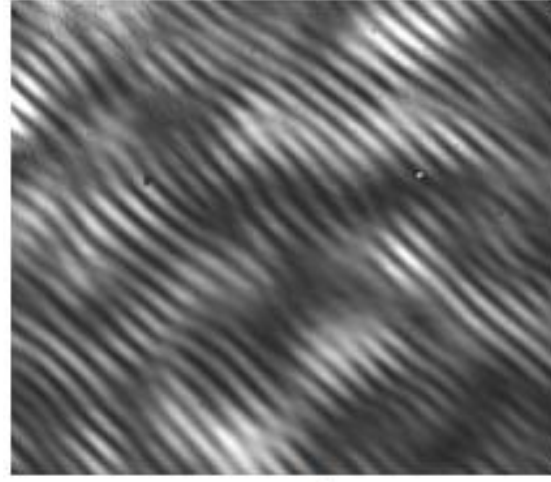

a

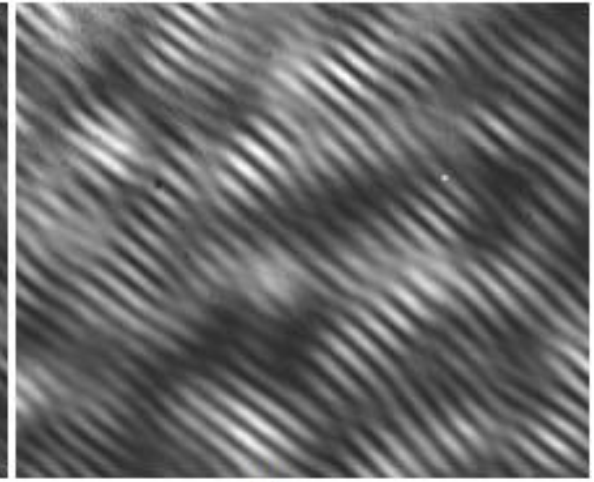

$\mathrm{b}$

Figure 3: Images of digital holograms recorded in a condition of loaded (a) and unloaded (b) interferometer

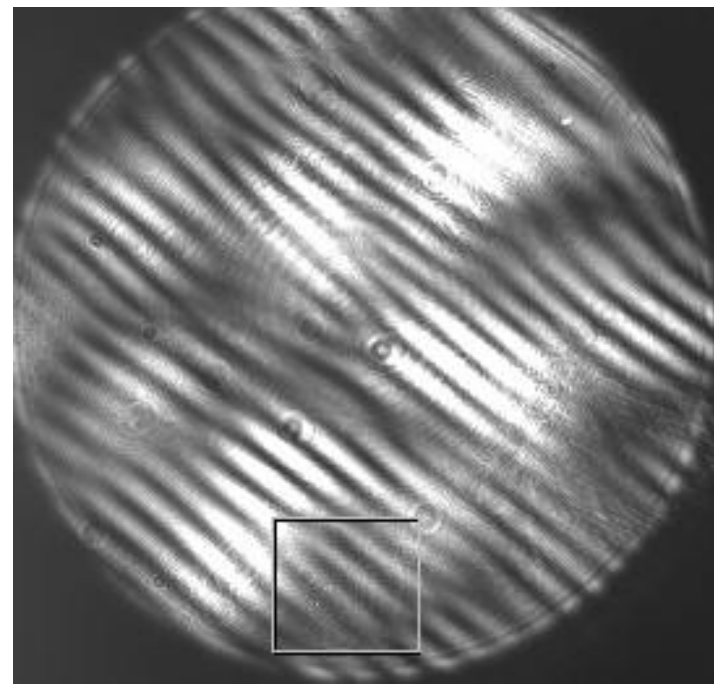

Figure 4: The digital hologram recorded by objective equipped camera.

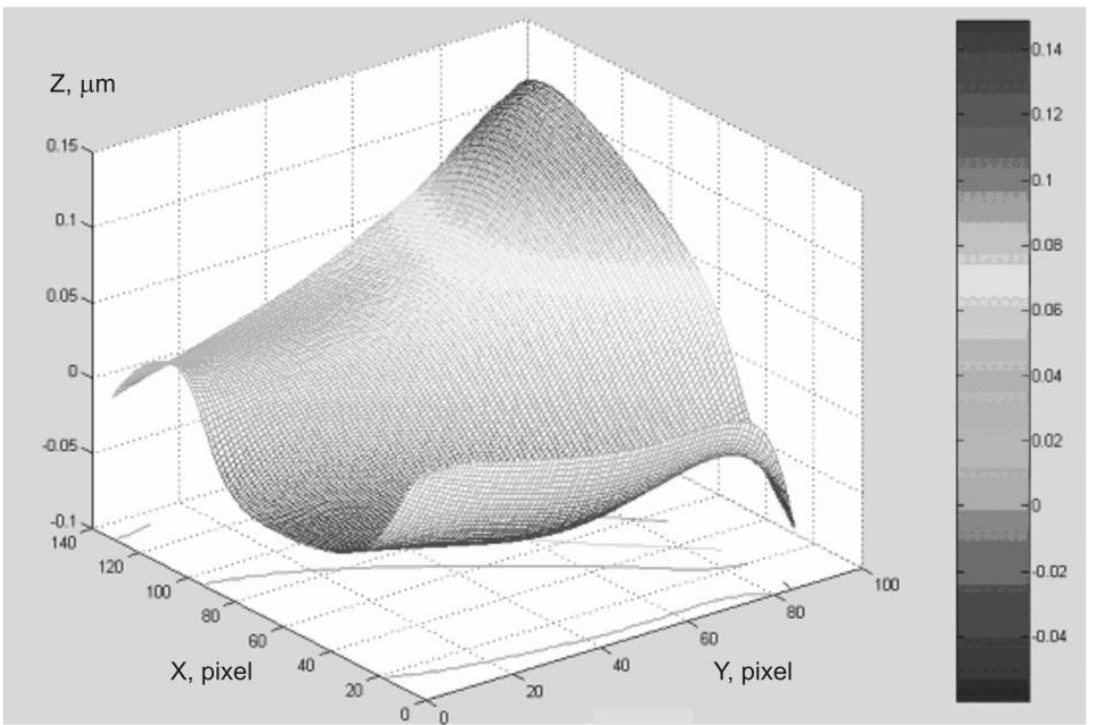

Figure 5: Interactive presentation of phase changes in the selected fragment of tested optical fiber section.

From this figure one can see that the phase incursion in laser radiation transmitted through the selected area in the fiber section in recalculation on optical path length is $0.22 \mathrm{~mm}$ at external dot loading on a cable of $3 \mathrm{mg}$. The accuracy of measurements along $\mathrm{z}$ axis was $5 \mathrm{~nm}$.

\section{Conclusion}

Thus the capacities of a digital double-exposed holographic intreferometry method and developed computational procedure for quantitative analysis of phase changes arising in radiation propagated in optical fiber at over small 
mechanical influences are shown. In our experiments the accuracy of measurements along $\mathrm{z}$ axis in recalculation of a phase on an optical path was $5 \mathrm{~nm}$. The above described method can be applied to diagnostics of optical splitters, connectors, focusing optics, welded assemblies of optical fibers. On the basis of the developed schemes the sensitive gauges of external influences can be created.

\section{References}

[1] U.Schnars, W.Jueptner. Digital Holography. - SpringerVerlag, Berlin (2005)

[2] D.Gabor. A new microscopic principle. Nature, 161, 777778 (1948)

[3] E.N.Leith, J.Upatnieks. Wavefront reconstruction with diffused illumination and threedimensional objects. J. Opt. Soc. Am., 54, 1295-1301 (1964)

[4] U.Schnars. Direct phase determination in hologram interferometry with use of digitally recorded holograms. J. Opt. Soc. Am. A, 11, 2011-2015 (1994)

[5] U.Schnars.DigitaleAufzeichnung und MathematischeRekonstruktion von Hologrammen in der Interferometrie. VDI-Fortschritt-Berichte. series 8. No 378. VDI, Dbsseldorf (1994)

[6] J.W.Goodman, R.W.Lawrence. Digital image formation from electronically detected holograms. Appl. Phys. Lett., 11, 77-79 (1967)

[7] R.W.Kronrod, N.S.Merzlyakov, L.P.Yaroslavskii. Reconstruction of a hologram with a computer. Sov. J. Tech. Phys., 17, 333-334 (1972)

[8] U.Schanrs, W.Juptner. Direct recording of holograms by a CCD target and numerical reconstruction. Appl. Opt., 33, 179-181 (1994)

[9] J.W.Goodman. Introduction to Fourier Optics. 2nd ed. McGraw-Hill, New York, (1996) 